\title{
Relative Velocity Fluctuations in Turbulent Rayleigh-Bénard Convection
}

\author{
P. Tong and Y. Shen \\ Department of Physics, Oklahoma State University, Stillwater, Oklahoma 74078
}

(Received 30 June 1992)

\begin{abstract}
High-Rayleigh-number turbulent convection has been studied using the technique of photoncorrelation homodyne spectroscopy to measure velocity differences at various length scales. The measured intensity correlation function $G(q, t, L)$ is found to be of the scaling form $G(q u(L) t)$, with $u(L) \sim L^{0.6}$ being the characteristic turbulent velocity at the length scale $L$ and $q$ the scattering vector. It is also found that the lower cutoff length $l_{c}$ of the scaling range obeys a power law $l_{c} \sim \mathrm{Ra}^{-0.3}$. The measured power-law exponents are in excellent agreement with theoretical predictions.
\end{abstract}

PACS numbers: 47.25.Qv, 05.40.+j, 42.25.-p

When a horizontal layer of fluid is heated from below, Rayleigh-Bénard convection will occur if the temperature difference $\Delta T$ across the layer exceeds a critical value $\Delta T_{c}$. The control parameter in thermal convection is the Rayleigh number $\mathrm{Ra}=\alpha g h^{3} \Delta T / v \chi$, with $\alpha$ being the thermal expansion coefficient, $g$ the gravitational acceleration, $h$ the thickness of the fluid layer, and $v$ and $\chi$ the kinematic viscosity and the thermal diffusivity of the fluid, respectively. In recent years much attention has focused on Rayleigh-Bénard convection both in the chaotic regime, where $\mathrm{Ra}$ slightly exceeds a critical Rayleigh number $\mathrm{Ra}_{c}$ (proportional to $\Delta T_{c}$ ), and in the turbulent regime, where $\mathrm{Ra} \gg \mathrm{Ra}_{c}$. In the latter case the temperature gradient across the fluid layer is concentrated in thin thermal boundary layers near upper and lower surfaces. The flow in the center of the cell is homogeneous and is believed to be characteristic of free-convection flow.

The recent discovery $[1,2]$ of scaling laws in the heat flux and temperature statistics in turbulent convection shed new light on the nature of convective turbulence. These experiments have stimulated considerable experimental [3] and theoretical [1,2,4-7] efforts, aimed at explaining the observed scaling laws in the temperature field. However, in contrast to the great number of temperature measurements, experimental information about the velocity field in turbulent convection is limited. In this Letter we report a light scattering study of high-Ra turbulent convection, from which the probability density function $P(V, l)$ of the velocity difference $V(l, t)$, between two points separated by a distance $l$, can be inferred. The experiment suggests that $P(V, l)$ has the form

$$
P(V, l)=Q(V / u(l)) / u(l),
$$

with the scaling velocity $u(l) \sim l^{0.6}$. This is in excellent agreement with the theoretical predictions by Procaccia and Zeitak [4] and L'vov [5].

A unique feature of convective turbulence is its interaction with the gravitational field at various length scales. Temperature is not a passive scalar in the system. This is absent in the normal barotropic turbulence such as that in a pipe flow. According to the Kolmogorov theory [8] of fully developed barotropic turbulence, turbulent kinetic energy is continuously transferred from the largest eddies of size $l_{0}$ to eddies of smaller sizes, until it dissipates when the size of the eddies becomes comparable to the viscous dissipation length $l_{d}$. In the inertial range, $l_{d}$ $<l<l_{0}$, the energy cascades at a constant rate $\epsilon$ without dissipation. As a consequence of the argument, the velocity difference $V(l, t)$ is expected to be scale invariant [9], and its probability density function $P(V, l)$ is a homogeneous function [10], as shown in Eq. (1). With a simple dimensional argument [8,9], one can show that the scaling velocity $u(l) \simeq(\epsilon l)^{1 / 3}$. For thermal convective turbulence, on the other hand, velocity fluctuations produce temperature fluctuations of various sizes, and eddies of different sizes can exchange different amounts of energy with the gravitational field. Therefore, the energy cascade rate $\epsilon(l)$ becomes $l$ dependent, which will be responsible for the departure from the Kolmogorov scaling of $u(l)$. It has been shown [4,5] that for convective turbulence, $u(l) \sim l^{3 / 5}$. Since the high-Ra convection provides an interesting variation of turbulence, direct measurement of the new exponent becomes fundamentally important in testing the general scaling argument for the description of turbulence.

In the experiment, to be described below, the technique of photon-correlation homodyne spectroscopy (HS) [11] is utilized to probe the instantaneous velocity difference $V(l, t)$. With the HS scheme, small seed particles in the fluid scatter light and follow the local flow. A photodetector records the scattered light intensity $I(t)$, which fluctuates due to the motion of the flowing particles. The output of the detector is therefore modulated at frequencies equal to differences in Doppler shifts of all particle pairs in the scattering volume. With the so-called homodyne method, one measures the intensity autocorrelation function [11] $g(t)=\left\langle I\left(t^{\prime}+t\right) I\left(t^{\prime}\right)\right\rangle /\langle I\rangle^{2}=1+b G(q, t, L)$, where $b$ is an instrumental constant which is chosen so that $G(t=0)=1$. The function $G(q, t, L)$ has the form [10]

$$
G(q, t, L)=\int_{0}^{L} d l h(l) \int_{-\infty}^{\infty} d V P(V, l) \cos (q V t),
$$

where $h(l)=2(1-l / L) / L$ is the number fraction of particle pairs separated by a distance $l$ in the thin scattering 
volume of length $L$, and $V(l, t)$ is the component of $\mathbf{V}(l, t)$ along the scattering vector $\mathbf{q}$. The amplitude of $\mathbf{q}$ is $q=(4 \pi / \lambda) \sin (\theta / 2)$, with $\theta$ being the scattering angle and $\lambda$ the wavelength of the light in the fluid. The function $G(q, t, L)$ yields information about the velocity differences in the $\mathbf{q}$ direction and at various scales $l$ (eddy sizes) up to $L$. If the velocity density function $P(V, l)$ has the form shown in Eq. (1), Eq. (2) then becomes [10]

$$
G(q t u(L))=\int_{0}^{L} d l h(l) F(q t u(l)),
$$

where $F(q t u(l))$ is the Fourier cosine transform of $Q(V / u(l))$. The HS technique has been used to study turbulent flows in a pipe and in a square tunnel $[10,12]$.

The convection cell used in the experiment was a vertical cylindrical cell with inner diameter $20 \mathrm{~cm}$. The upper and lower plates were made of brass, and the sidewall of the cell was a cylindrical ring made of transparent Plexiglas to admit the incident light and observe the scattering. Two cylindrical rings with heights 20 and $6.6 \mathrm{~cm}$ were used respectively to extend the accessible range of $\mathrm{Ra}$. The temperature of the upper plate was regulated within $0.05^{\circ} \mathrm{C}$ by passing cold water through the cooling chamber fitted on the top of the plate. The lower plate was heated uniformly at a constant rate with an electric heating wire imbedded on the backside of the plate. The electric current was provided by a regulated dc power supply with $0.1 \%$ long-term stability. The temperature difference $\Delta T$ between the two plates was measured by two thermistors imbedded in the two plates. The vertical heat flux through the cell was determined from the power required to keep the lower plate at a constant temperature. Our measurements of the Nusselt number $\mathrm{Nu}$ (the normalized heat flux) as a function of $\mathrm{Ra}$ are well described by the power law $\mathrm{Nu}=0.16 \mathrm{Ra}^{\beta}$ when $\mathrm{Ra}$ is in the range between $4 \times 10^{7}$ and $10^{10}$. The measured exponent $\beta=0.29 \pm 0.015$, which agrees well with previous measurements $[1,3,13]$.

The cell was filled with water seeded with polystyrene latex spheres of diameter $0.14 \mu \mathrm{m}$. The volume fraction of the seed particles was $\sim 10^{-4}$. At this particle concentration, the particle mean spacing is much larger than their diameter (dilute solution) but much smaller than the smallest turbulent scale (sufficient sampling). Measurements of $g(t)$ were performed with a standard light scattering apparatus and a multichannel correlator. The incident beam from a $3-\mathrm{W}$ argon-ion laser $\left(\lambda_{0}=514.5\right.$ $\mathrm{nm}$ ) traversed through the center of the cell. The focused incident beam had a typical diameter of $0.1 \mathrm{~mm}$. The laser beam was then imaged with three different magnifications onto a slit of variable width from 0.1 to $1.2 \mathrm{~mm}$. Three lenses with different focal lengths were used in order to vary the magnification. With the three magnifications and the range of the slit widths, the real length $L$ of the thin cylindrical scattering volume viewed by a photomultiplier was varied between 0.1 and $17 \mathrm{~mm}$. The length $L$ determines the maximum measurable eddy size,
$l=L$, to which $g(t)$ is sensitive. Light passing through the slit fell on the photomultiplier, which recorded the time-varying intensity $I(t)$. The photomultiplier was located far behind the slit $(\sim 80 \mathrm{~cm})$, so that light was collected from roughly one coherence area. Measurements were made at the scattering angle $\theta=90^{\circ}$.

To characterize the nonexponential decaying function $G(q, t, L)$, a decay rate $\Gamma(L)$ is defined as $\Gamma^{-1}(L)$ $=\int_{0}^{\infty} d t G(q, t, L)$. This definition of $\Gamma(L)$ emphasizes the initial decay of $G(q, t, L)$ governed by $L$. With a simple dimensional argument one can show that $\Gamma(L)$ $\simeq q u(L)$, where $u(L)$ is the characteristic eddy velocity at scale $L$. Several hundred correlation functions have been measured as a function of $L$ and $\mathrm{Ra}$. In the absence of convection $(\mathrm{Ra}=0), G(q, t, L)$ still decays because of the Brownian motion of the seed particles. The Brownian motion contributes a factor $\exp \left(-2 D q^{2} t\right)$ to $G(q, t, L)$, where $D$ is the diffusion constant of the particles [11]. The Brownian decay rate $2 D q^{2}$ has been subtracted from the measured $\Gamma(L)$, to be discussed below. This correction is especially important for the low-Ra data, because the flow-induced decay rate in this case is comparable to the Brownian decay rate.

The measured $G(q, t, L)$ is found to be of scaling form $G(\kappa)$, with $\kappa=\Gamma(L) t \simeq q t u(L) . \quad \log -\log$ plots of $G(q$, $t, L)$ for various values of $L$ and $\mathrm{Ra}$ can be brought into coincidence by sliding them horizontally with respect to each other. The decay time $\Gamma^{-1}(L)$ defined above quantitatively characterizes the amount of the horizontal translation that is required to bring the correlation functions into coincidence. Figure 1 shows typical $G(\kappa)$ as a function of $\kappa$ for various values of $\mathrm{Ra}$ and $L$. As shown in Eq. (3), a scaling $G(q t u(L))$ implies that the probability density function $P(V, l)$ has the form shown in Eq. (1). Our measurements thus suggest that the functional form

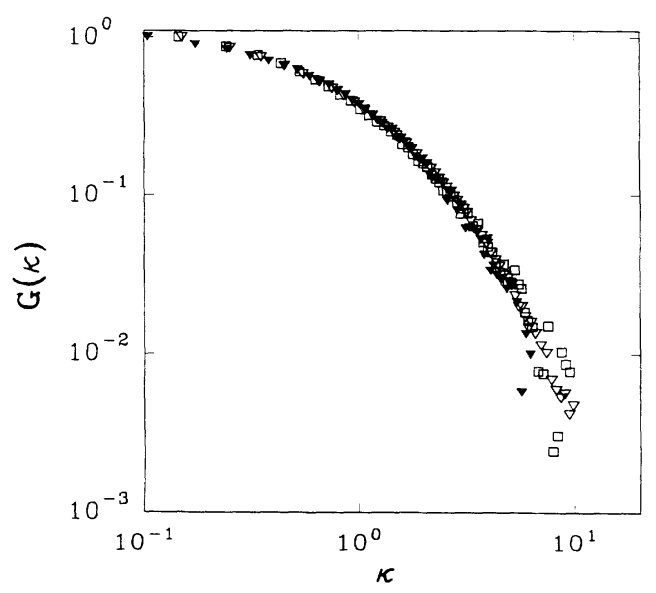

FIG. 1. The measured correlation function $G(\kappa)$ vs $\kappa$ $=\Gamma(L) t=q t u(L)$. The experimental conditions are $L=4.1$ $\mathrm{mm}, \mathrm{Ra}=9.1 \times 10^{9}$ (solid triangles); $L=6.8 \mathrm{~mm}, \mathrm{Ra}=9.1 \times 10^{9}$ (open squares); and $L=13.5 \mathrm{~mm}, \mathrm{Ra}=3.4 \times 10^{9}$ (open triangles). 


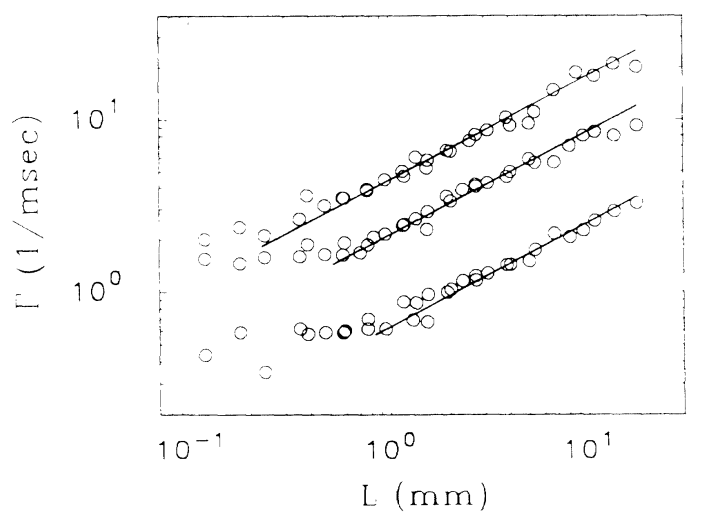

FIG. 2. Variations of the decay rate $\Gamma(L)$ with the length $L$ measured at $\mathrm{Ra}=9.1 \times 10^{9}$ (top curve), $\mathrm{Ra}=2.2 \times 10^{9}$ (middle curve), and $\mathrm{Ra}=3.7 \times 10^{8}$ (bottom curve). The solid lines are power-law fits: $4.47 L^{0.6}[1 / \mathrm{ms}]$ (top line), $2.14 L^{0.6}[1 / \mathrm{ms}]$ (middle line), and $0.63 L^{0.6}[1 / \mathrm{ms}]$ (bottom line).

of $P(V, l)$ and, hence, the turbulent structure are invariant in $\mathrm{Ra}$, and the statistical properties of $V(l, t)$ over varying length scales become identical under an appropriate scaling of velocities.

The scale dependence of the scaling velocity is examined by measuring the $L$ dependence of $\Gamma(L)$. Figure 2 shows the measured $\Gamma(L)$ as a function of $L$ at three different values of $\mathrm{Ra}$. There are three important features in this plot.

First, the decay rate $\Gamma(L)$ as a function of $L$ obeys a power law $\Gamma(L) \sim L^{\xi}$ [i.e., $u(L) \sim L^{\xi}$ ]. From the straight-line segment (solid lines in Fig. 2), one finds the exponent $\xi=0.60 \pm 0.02$. The power-law behavior of $\Gamma(L)$ is found when $5 \times 10^{7} \leq \mathrm{Ra} \leq 10^{10}$. The velocity component measured in the experiment is perpendicular to the direction of the gravity. Our experiment, therefore, directly confirms the theoretical prediction $[4,5]$ that $\xi=\frac{3}{5}$. The exponent $\xi$ was also inferred from a temperature measurement by Wu et al. [2,5].

Second, Fig. 2 shows that there is a cutoff length $l_{c}$, below which $\Gamma(L)$ levels off. It is seen that the cutoff length $l_{c}$ decreases as $\mathrm{Ra}$ is increased. The measured $l_{c}$ as a function of Ra is shown in Fig. 3 (solid circles). The result can be described by the power law (lower solid line) $l_{c}=34.3 \mathrm{Ra}^{-\gamma}[\mathrm{cm}]$. The exponent is $\gamma=0.30$ \pm 0.03 , which agrees with the theoretical prediction [5] that $l_{c} \sim \mathrm{Ra}^{-5 / 16}$. The scatter in the data is due to the uncertainty in determining $l_{c}$ from the plot of $\Gamma(L)$ vs $L$. Procaccia and Zeitak [4] have proposed that there may be a length scale, $l_{b} \simeq h \mathrm{Ra}^{-3 / 28}$, below which a crossover to Kolmogorov exponents is expected. However, such a length scale is not observed in our measurements of $\Gamma(L)$. This finding supports L'vov's argument that in high-Ra convective turbulence the entropy flux at various length scales is constant $[5,14]$. Notice that below $l_{c}$ the measured $\Gamma(L)$ becomes $L$ independent. This is an interest-

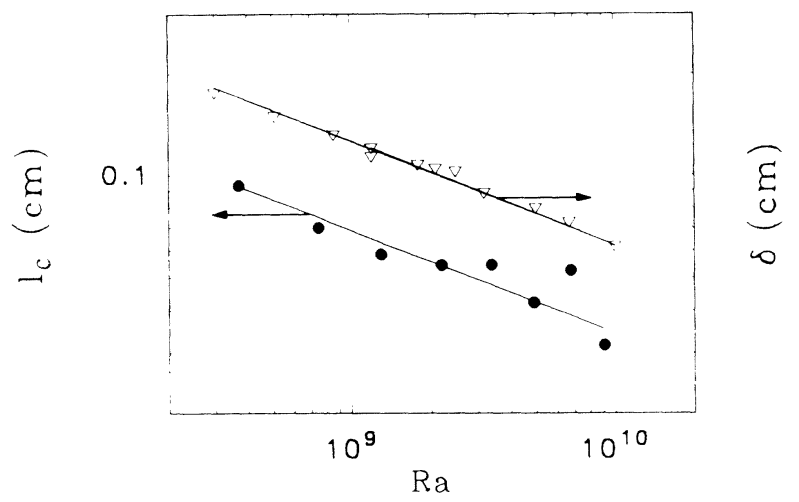

FIG. 3. Variations of the cutoff length $l_{c}$ (solid circles) and the thermal boundary layer thickness $\delta$ (open triangles) as a function of $\mathrm{Ra}$. The upper solid line is a power-law fit $63.0 \mathrm{Ra}^{-0.3}[\mathrm{~cm}]$, and the lower solid line is $34.3 \mathrm{Ra}^{-0.3}[\mathrm{~cm}]$.

ing phenomenon which will be discussed elsewhere [15].

We now address an important issue concerned with the relationship between coherent structures and the velocity scaling. It was found $[3,16]$ that the main coherent structures in the convective turbulence are plumes, which consist of a column of buoyant fluid emanating from the boundary layer and culminating in a cap, and thermals, which are free plumes not connected to the boundary layer. Because they are generated from the boundary layers, the smallest size of these coherent structures (the diameter of the thermal plumes, see Fig. 1 of Ref. [16]) is naturally controlled by the thermal boundary layer thickness $\delta$. In Fig. 3 we plot $\delta$ (open triangles), which is obtained from the measured Nusselt number $\mathrm{Nu}$, using the relation $\delta=h / 2 \mathrm{Nu}$. The data are well described by the power law $\delta=63.0 \mathrm{Ra}^{-0.3}$ when $5 \times 10^{7} \leq \mathrm{Ra} \leq 10^{10}$. It turns out that the value of our $\delta$ is very close to that measured directly from the temperature profile in a similar convection cell [16]. It is seen from Fig. 3 that the two length scales, $l_{c}$ and $\delta$, have similar power-law behavior. The values of the two exponents are the same within our experimental uncertainties. The numerical values of the two length scales only differ by a factor of 2 . We therefore conclude that the measured cutoff length $l_{c}$ in the velocity scaling is determined by the smallest size of the coherent structures in the core region of turbulent convection.

The third feature in Fig. 2 is that at a fixed $L$, the measured $\Gamma(L)$ [or equivalently $u(L)$ ] is also a function of Ra. In Fig. 4(a) the Reynolds number $\operatorname{Re}(L)$ (the normalized velocity) is plotted as a function of $\mathrm{Ra}$ for two different values of $L$, where $\operatorname{Re}(L)=h \Gamma(L) / q(v \chi)^{1 / 2}$ $\simeq h u(L) /(v \chi)^{1 / 2}$. The measured $\operatorname{Re}(L)$ as a function of $\mathrm{Ra}$ is well described by the power law (solid lines) $\operatorname{Re}(L) \sim \operatorname{Ra}^{0.67}$. The uncertainty for the exponent is \pm 0.03 . Combining Figs. 2 and $4(\mathrm{a})$, we have $\operatorname{Re}(L)$ $\sim L^{0.6} \mathrm{Ra}^{0.67}$. This result appears to be different from the 


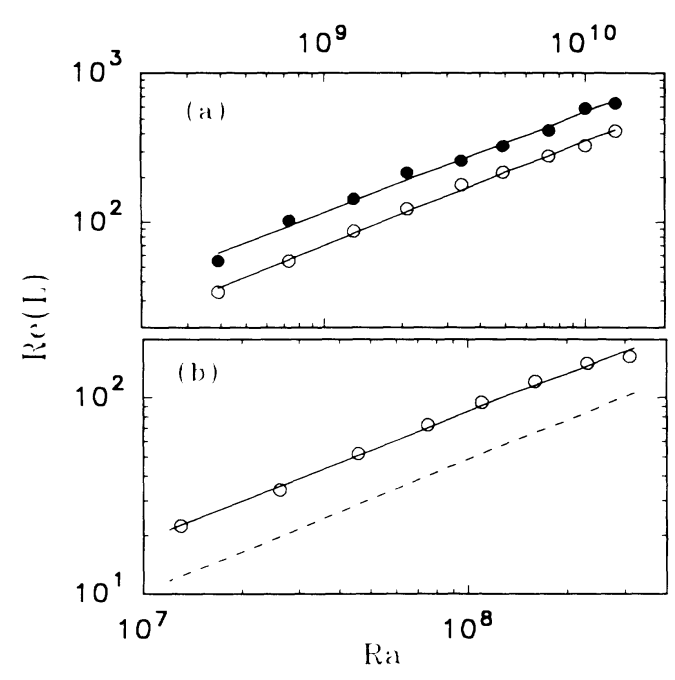

FIG. 4. (a) The measured Reynolds number $\operatorname{Re}(L)$ as a function of $\mathrm{Ra}$ at $L=13.5 \mathrm{~mm}$ (solid circles) and $L=5.4 \mathrm{~mm}$ (open circles) in the $h=20 \mathrm{~cm}$ cell. The solid lines are the power-law fits by $\operatorname{Re}(L) \sim \mathrm{Ra}^{0.67}$. (b) The measured $\operatorname{Re}(L)$ at $L=13.5 \mathrm{~mm}$ (open circles) in the cell with $h=6.6 \mathrm{~cm}$. The solid line is the power-law fit by $\operatorname{Re}(L) \sim \mathrm{Ra}^{0.67}$. The dashed line shows the extrapolation of the measured $\operatorname{Re}(L)$ in the $h=20 \mathrm{~cm}$ cell with the $h^{-3 / 5}$ correction.

theoretical prediction [5] that $\operatorname{Re}(L) \simeq(L / h)^{3 / 5} \mathrm{Ra}^{1 / 2}$. One possible reason for this deviation from the predicted scaling exponent in $\mathrm{Ra}$ is the anisotropic nature of convective turbulence at length scales comparable to the size $h$ of the cell. Fluctuations of the velocity difference $V(l, t)$, between two points separated by $l \simeq h$, in the direction parallel to the gravity are certainly different from those in the perpendicular direction. The above prediction, on the other hand, is obtained by assuming the velocity fluctuations are isotropic when $L \simeq h$. Put in another way, the local Rayleigh number $\operatorname{Ra}(L)$ should be used as a scaling variable rather than the global Rayleigh number $\mathrm{Ra}$. This argument can explain the observation that the $h$ dependence of the measured $\operatorname{Re}(L)$ is also different from the theoretical prediction. Figure 4(b) shows the measured $\operatorname{Re}(L)$ as a function of $\mathrm{Ra}$ at $L=13.5 \mathrm{~mm}$ in the $h=6.6 \mathrm{~cm}$ cell (open circles). The solid line in the plot is the power-law fit $\operatorname{Re}(L) \sim \mathrm{Ra}^{0.67}$. The dashed line shows the extrapolation of the measured
$\operatorname{Re}(L)$ in the $h=20 \mathrm{~cm}$ cell with the predicted $h^{-3 / 5}$ correction. Figures $4(\mathrm{a})$ and $4(\mathrm{~b})$ show that $\operatorname{Re}(L)$ is larger for smaller values of $h$ when the other experimental conditions are kept unchanged. However, the data fail to show the predicted $h^{-3 / 5}$ dependence.

We thank W. I. Goldburg, X.-Z. Wu, B. J. Ackerson, T. A. Witten, and D. S. Cannell for stimulating conversations. Thanks are also due to M. Lucas and his team for fabricating the experimental cells and to K. Suhm for the experimental assistance at the early stage of the experiment. Acknowledgment is made to the Donors of The Petroleum Research Fund, administered by the American Chemical Society, for the partial support of this work.

[1] F. Heslot, B. Castaing, and A. Libchaber, Phys. Rev. A 36, 5870 (1987); B. Castaing et al., J. Fluid Mech. 204, 1 (1989).

[2] X.-Z. Wu, L. Kadannoff, A. Libchaber, and M. Sano, Phys. Rev. Lett. 64, 2140 (1990).

[3] T. H. Solomon and J. P. Gollub, Phys. Rev. Lett. 64, 2382 (1990); Phys. Rev. A 43, 6683 (1991).

[4] I. Procaccia and R. Zeitak, Phys. Rev. Lett. 62, 2128 (1989).

[5] V. S. L'vov, Phys. Rev. Lett. 67, 687 (1991).

[6] Zhen-Su She, Phys. Fluids A 1, 911 (1989).

[7] B. I. Shraiman and E. D. Siggia, Phys. Rev. A 42, 3650 (1990).

[8] A. N. Kolmogorov, C. R. Dokl. Acad. Sci. USSR 30, 301 (1941); 31, 538 (1941).

[9] U. Frisch, P. Sulem, and M. Nelkin, J. Fluid Mech. 87, 719 (1978).

[10] P. Tong, W. I. Goldburg, C. K. Chan, and A. Sirivat, Phys. Rev. A 37, 2125 (1988).

[11] B. J. Berne and R. Pecora, Dynamic Light Scattering (Wiley, New York, 1976).

[12] P. Tong and W. I. Goldburg, Phys. Fluids 31, 2841 (1988); H. K. Pak, W. I. Goldburg, and A. Sirivat, Fluid Dyn. Res. 8, 19 (1991).

[13] H. Tanaka and H. Miyata, Int. J. Heat Mass Transfer 23, 1273 (1980).

[14] V. S. L'vov and G. E. Falkovich, Weizmann Institute of Science Report No. WIS-91/7/Mar-PH, 1991 (unpublished).

[15] P. Tong et al. (to be published).

[16] G. Zocchi, E. Moses, and A. Libchaber, Physica (Amsterdam) 166A, 387 (1990). 